\title{
Hidroxiapatita sintetizada a partir del reciclaje de cascaron de huevo
}

\section{Hydroxyapatite synthesized from the recycling of egg shells}

ENRÍQUEZ-PÉREZ, Ma. Angelest’’, CASTREJÓN-SÁNCHEZ, Víctor Hugo*’, ROSALESDAVALOS, Jaime" y DÍAZ-CAMACHO, Francisco Javier A.'

'Tecnológico de Estudios Superiores de Jocotitlán, Departamento de Ingeniería en Materiales, México.

"Tecnológico de Estudios Superiores de Jocotitlán, Departamento de Ingeniería Mecatrónica, México.

ID 1er Autor: Ma. Angeles, Enríquez-Pérez / ORC ID: 0000-0002-2280-0661, Researcher ID Thomson: H-9399-2018, CVU CONACYT ID: 1T16E134

ID 1er Coautor: Víctor Hugo, Castrejón-Sánchez / ORC ID: 0000-0002-0112-5388, Researcher ID Thomson: C-90772015, CVU CONACYT ID: 235470

ID 2do Coautor: Jaime, Rosales-Davalos / ORC ID: 0000-0002-9059-6093, CVU CONACYT ID: 812961

ID 3er Coautor: Francisco Javier A., Díaz-Camacho / ORC ID: 0000-0002-2878-2281

DOI: $10.35429 /$ JOTI.2020.14.4.1.6

Recibido Julio 10, 2020; Aceptado Diciembre 30, 2020

\section{Resumen}

Existen diferentes tipos de hidroxiapatitas (HAp), por lo que sus usos se han extendido no solo en el área de biomateriales, en catálisis empieza a ser analizado su posible uso. Sus propiedades dependen de la relación atómica $\mathrm{Ca} / \mathrm{P}$, donde el tipo de síntesis y materia prima influyen en esta relación. Por esta razón, en este trabajo se sintetizo HA a partir del desecho del cascarón de huevo, su estructura está compuesta en un $90 \%$ de $\mathrm{CaCO}_{3}$, se impregno el fosforo, el material obtenido se calcino a 800 ${ }^{\circ} \mathrm{C}$, variando el tiempo 2,4 y $6 \mathrm{H}$. Se encontró que el material obtenido presenta los grupos funcionales característicos de las $\mathrm{HA}$, la relación $\mathrm{Ca} / \mathrm{P}$ se obtuvo por EDS, oscila entre 4.8 a 44, lo que indica que se tiene una HA con una gran cantidad de grupos carbonatos.

Hidroxiapatita, Relación atómica, Catálisis

\begin{abstract}
There are different types of hydroxyapatites (HAp) which is why their uses have extended not only in the area of biomaterials, but in catalysis their possible use begins to be analyzed. Its properties depend on the $\mathrm{Ca} / \mathrm{P}$ atomic ratio, where the type of synthesis and raw material influence this relationship. For this reason, in this work HA was synthesized from the waste of the egg shell, its structure is made up to $90 \% \mathrm{CaCO}_{3}$, phosphorus was impregnated, the material obtained was calcined at $800^{\circ} \mathrm{C}$, varying the time 2, 4 and $6 \mathrm{H}$. The material obtained was found to have characteristic functional groups of HAp, the $\mathrm{Ca} / \mathrm{P}$ ratio was obtained byEDS, ranging from 4.8 to 44 , indicating that you have HA with many carbonate groups.
\end{abstract}

Hydroxyapatite, Atomic radio, Catalysis

Citación: ENRÍQUEZ-PÉREZ, Ma. Angeles, CASTREJÓN-SÁNCHEZ, Víctor Hugo, ROSALES-DAVALOS, Jaime y DÍAZ-CAMACHO, Francisco Javier A. Hidroxiapatita sintetizada a partir del reciclaje de cascaron de huevo. Revista de Invención Técnica. 2020. 4-14:1-6. 


\section{Introducción}

Dentro de la familia de los materiales cerámicos, destacan los derivados del fosfato de calcio, debido a su biocompatibilidad con el cuerpo humano (García \& Reyes, 2006); entre ellos se encuentra la hidroxiapatita, que puede ser de origen natural o sintética, es usada en las áreas de Odontología, Ortopedia, cirugía plástica y Cirugía Maxilofacial (Gomes, Santos, Neves, \& Menezes, 2019; Rivera, y otros, 2004)

La hidroxiapatita (HAp) pura estequiométrica, está formada por átomos de calcio, fósforo e hidrógeno (Rivera, y otros, 2004), de acuerdo con la fórmula: $\mathrm{Ca}_{10}\left(\mathrm{PO}_{4}\right)_{6}(\mathrm{HO})_{2}$. Se conoce como apatita, debido a que se pueden formar diferentes clases de compuestos con una gran variedad de composiciones con formula general:

$\mathrm{X}_{3} \mathrm{Y}_{2}\left(\mathrm{TO}_{4}\right) \mathrm{Z}$

\section{Donde:}

$\mathrm{X}$ o $\mathrm{Y}=\mathrm{Ca} . \mathrm{Sr}, \mathrm{Ba}, \mathrm{Re}, \mathrm{Pb}, \mathrm{U}, \mathrm{Mn}$ y raramente $\mathrm{Na}$.

$$
\mathrm{T}=\mathrm{P}, \mathrm{As}, \mathrm{V}, \mathrm{Si}, \mathrm{S} \text { y } \mathrm{C}\left(\mathrm{como} \mathrm{CO}_{3}\right)
$$

$\mathrm{Z}=\mathrm{F}, \mathrm{Cl}, \mathrm{OH}^{-}$y $\mathrm{O}$ (Coreño, Mújica, \& Hernández, 2010)

Estas combinaciones hacen que la HAp un material atractivo no sólo para el área médica, sino que sus usos se han ido extendido en la industria de catálisis, fertilizantes, farmacéutica y en procesos de tratamiento de aguas residuales (Gomes, Santos, Neves, \& Menezes, 2019). Un factor importante entre las distintas HAp es su relación atómica $\mathrm{Ca} / \mathrm{P}$, debido a que influye en sus propiedades. Una HAp estequiométrica es la que tienen una relación de 1.67 (Dai, Tan, Zhu, Sun, \& Wang, 2018), valores menores reportan una hidroxiapatita carente de $\mathrm{Ca}$ y valores mayores posee una gran cantidad de carbonatos (Londoño, Echavarría, \& De La Calle, 2006). Esta relación, está relacionada básicamente con dos factores:

- Tipo de síntesis, por lo general química, se encuentran métodos como hidrotermal, precipitación y sol-gel.
- Tipo de materia prima, puede sintético o fuentes naturales (huesos: de pescado, porcinos u bovinos, corales, escamas, cascaron de huevo) (Jin, Chen, Cheng, Wang, \& Hu, 2015),(Sánchez, Berini, \& Gay, 1993).

Cada día, se generan desechos sólidos debido a las actividades humanas; es por eso, que la síntesis verde es un método alternativo (Reyes, 2002), por ejemplo la HAp puede ser obtenida a partir de fuentes naturales, siendo un proceso de síntesis de bajo costo, en comparación de la HAp sintetizada químicamente, donde los parámetros como temperatura, $\mathrm{pH}$ y pureza de los reactivos iniciales son fundamentales $\mathrm{y}$ deben ser constantemente controlados para obtener HAp estequiométrica de alta calidad, elevando los costos y tiempo requerido en su síntesis (Akram, Ahmed, Shakir, Ibrahim, \& Hussain, 2014). Sin embargo las fuentes naturales cumplen dos funciones básicas; ahorro económico y reducción de desechos, por ejemplo México ocupa el $4^{\text {to }}$ lugar a nivel mundial en producción de huevo, se calcula que se procesan alrededor de 45 mil millones de huevos, se tiene el consumo de huevo per cápita más alto en el mundo, con un consumo promedio de 345 huevos por persona cada año, casi 1 huevo diario (García S. , 2019).

La cascara del huevo, está compuesta por carbonato de calcio (94\%), fosfato de calcio $(1 \%)$ y trazas de otros minerales (1\%) (Camacho Díaz, y otros, 2018), al ser calcinada a se obtiene $\mathrm{CaO}$, que es empleado como precursor de calcio en la síntesis y se le adiciona una solución de fosfato de calcio $\left(\mathrm{Ca}_{3}\left(\mathrm{PO}_{4}\right)_{2}\right)$ donde las cantidades utilizadas no son perjudiciales para el medio ambiente y se obtiene una HAp de alta calidad (Rivera, Fetter, \& Bosch, 2011); en este trabajo se reporta la síntesis de la Hidroxiapatita a partir del reciclaje del cascaron de huevo, se impregnaron los fosfatos con ácido fosfórico, el material obtenido se calcina a $800{ }^{\circ} \mathrm{C}$, se varia el tiempo del tratamiento a 2,4 y 6 horas, respectivamente; posteriormente, el material se caracteriza para determinar el tipo de hidroxiapatita que se obtiene. El material obtenido, se pretende usarlo en fotocatálisis para eliminación de colorantes orgánicos. 


\section{Metodología}

\section{Reactivos}

Peróxido de hidrogeno (90\%), Ácido fosfórico (85\%) adquiridos en Sigma-Aldrich y agua destilada.

\section{Síntesis de HAp}

Se hizo la recolección de cascaron de huevo (900 g), se lavó con agua corriente y después con peróxido de hidrogeno al $70 \%$, después se secó a $80{ }^{\circ} \mathrm{C}$ por $48 \mathrm{~h}$. El material seco se trituro con un mortero de agata y se pasó por un tamiz \#80, posteriormente se impregnó el material con ácido fosfórico $1 \mathrm{M}$ por $5 \mathrm{~h}$, después se hacen lavados con agua destilada hasta un $\mathrm{pH}$ neutro. El material se calcina a $800^{\circ} \mathrm{C}$, los tiempos de calcinación se establecieron en 2, 4 y $6 \mathrm{~h}$. El cerámico obtenido se muele en un mortero de agata y se pasó por un tamiz \#80.

\section{Caracterización de HAp}

\section{Microscopia Electrónica de barrido}

Para conocer la morfología del material obtenido, se usó un Microscopio Electrónico de Barrido (Jeol IT-100) acoplado a un a microsonda de rayos $\mathrm{X}$ (Bruker) para análisis elemental. Se realizaron micrografías con amplificaciones de 1000 y 3000x.

Se realizó un análisis elemental al material obtenido, sobre la superficie para obtener información acerca de los elementos y la relación $\mathrm{Ca} / \mathrm{P}$ de la $\mathrm{HAp}$ por espectroscopia de dispersión de energía.

\section{Espectroscopia FTIR}

Para saber los grupos funcionales presentes en la HAp, se utilizó un espectrofotómetro PerkinElmer FT modelo 2000 en modo ATR, con un intervalo de 4000 a $500 \mathrm{~cm}^{-1}$, el análisis se realizó directamente sobre la muestra.

El espectro de IR representa en el eje de las abscisas la longitud de onda $\left(\mathrm{cm}^{-1}\right)$ contra el porciento de transmitancia (T\%).

\section{Espectroscopia Raman}

Para determinar las fases cristalinas de las muestras, se usó un micro Raman modelo XploraPlus Jobin Yvon Horiba se usa un láser de estado sólido $(\lambda=532 \mathrm{~nm})$, la potencia máxima es de $25 \mathrm{~mW}$, se utiliza una lente de 50x para enfocar y colectar la luz dispersada. La potencia sobre la superficie de la muestra es del $10 \%$ de la potencia nominal. Se cuenta con una rejilla de difracción de 1200 líneas por milímetro, se promediaron 100 adquisiciones de $1 \mathrm{~s}$ cada una.

\section{Resultados}

\section{Microscopia Electrónica de barrido}

Para estudiar el efecto del tiempo (en h) en la relación $\mathrm{Ca} / \mathrm{P}$, las muestras se trataron térmicamente a $800^{\circ} \mathrm{C}$ por 2,4 y 6 horas, respectivamente.

En la figura 1a se muestra la micrografía de la HAp sin tratamiento térmico, donde se puede apreciar una morfología porosa con algunos agregados cristalinos sin orden, al aplicar el tratamiento térmico e ir aumentando el tiempo, por ejemplo a $2 \mathrm{~h}$ (figura 1b), estos agregados son más densos y están distribuidos de forma homogénea, pero al ir aumentando el tiempo del tratamiento existe un cambio en la distribución, a mayor tiempo hay una separación entre los aglomerados que a tiempos menores tienden a ser homogéneos (ver figura 1).

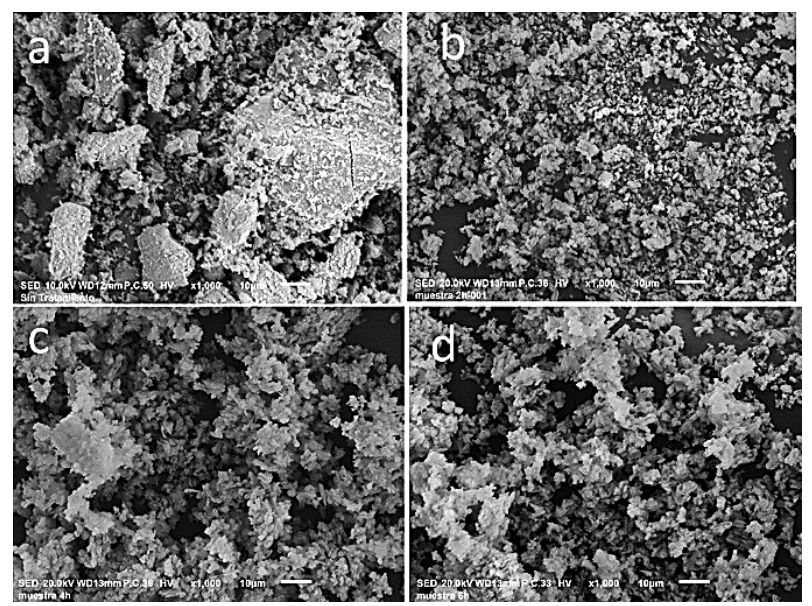

Figura 1 Micrografía de HAp a) sin tratamiento, con tratamiento térmico a: b) $2 \mathrm{~h}, \mathrm{c}$ ) $4 \mathrm{~h}$ y d) $6 \mathrm{~h}$

Fuente: Elaboración propia 


\section{Análisis elemental por EDAX}

La Tabla 1, muestra la relación atómica de los elementos que componen a la HAp como función del tiempo del tratamiento térmico (TT), el material obtenido presenta trazas de magnesio y no hay una relación en el tiempo del tratamiento. Se obtienen valores mayores a los reportados con la HAp estequiometria, a un tiempo menor se obtiene un valor de $4.9 \%$ at., Rivera, et al. (2011) reporto que cuando se tienen valores mayores de 1.67 indica que la apatita obtenida está formada en su mayoría por carbonatos y reporta además que se caracteriza por la presencia de trazas de $\mathrm{Mg}$, tal y como se encontró en las muestras analizadas.

\begin{tabular}{|c|c|c|c|c|c|c|}
\hline TT (H) & C & $\mathbf{O}$ & $\mathrm{Ca}$ & $\mathbf{P}$ & $\mathrm{Ca} / \mathrm{P}$ & $\mathbf{M g}$ \\
\hline 2 & 27.6 & 54.3 & 15.9 & 3.3 & 4.9 & 0.69 \\
\hline 4 & 18.1 & 55.8 & 23.0 & 0.81 & 28 & 0.35 \\
\hline 6 & 11.2 & 60.7 & 25.3 & 2.3 & 11 & 0.24 \\
\hline
\end{tabular}

Tabla 1 Relación atómica vs tiempo del tratamiento térmico.

Fuente: Elaboración propia

\section{FTIR}

En la Figura 2 se presentan los espectros de infrarrojo de las muestras de HAp. Sin tratamiento térmico, los picos en 1426 y $876 \mathrm{~cm}^{-}$ ${ }^{1}$ indican la presencia del grupo carbonato y las bandas características del grupo fosfato se observan en 714 y $540 \mathrm{~cm}^{-1}$, estas absorciones corresponden a la HAp, reportados previamente en la literatura (Fragoso, y otros, 2018). Cuando se da tratamiento térmico, en todos los tiempos aparece una banda a $3644 \mathrm{~cm}^{-1}$ corresponde a una absorción de alargamiento de grupos $\mathrm{OH}$ de la estructura de la HAp (Rivera, Fetter, \& Bosch, 2011), se observan las bandas características del fosfato a 1048 y $670 \mathrm{~cm}^{-1}$. Las absorciones a $1738,1450,1378,982$ y $874 \mathrm{~cm}^{-1}$ corresponden al grupo $\mathrm{CO}_{3}^{-2}$, las posiciones de estos picos han sido previamente reportadas (Pleshko, Boskey, \& Mendelsoh, 1991). Algunos autores (Rivera, Fetter, \& Bosch, 2011) (Pleshko, Boskey , \& Mendelsoh, 1991), han reportado que cuando se presentan estas últimas absorciones, indican que el material posee una mayor cantidad de carbonatos, lo cual concuerda con el análisis elemental realizado al material obtenido.

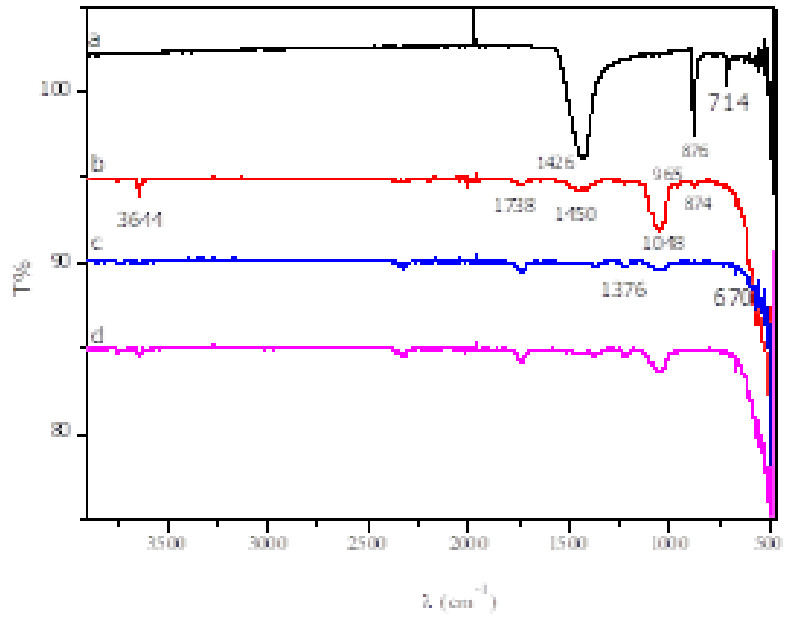

Figura 2 Espectro de FTIR de HAp; a) sin tratamiento, con tratamiento térmico: b) 2h, c) $4 \mathrm{~h}$ y d) $6 \mathrm{~h}$.

Fuente: Elaboración propia

\section{Espectroscopia microRaman}

En la figura 3, se muestran espectros Raman de la HAp, se analizan las señales en el rango de los 400 a los $1800 \mathrm{~cm}^{-1}$, debido a que es la región espectral del material obtenido, cuando el material se tratado térmicamente a un tiempo de 2 y $4 \mathrm{~h}$ la señal de $964 \mathrm{~cm}^{-1}\left(v_{1} \mathrm{PO}_{4}{ }^{3-}\right)$ es una banda de fosfato típica asociada a la apatita carbonatada (Ruíz, y otros, 2018), la señal de $1078 \mathrm{~cm}^{-1}\left(\mathrm{v}_{2} \mathrm{PO}_{4}{ }^{3-}\right)$ se atribuyen a los modos de flexión del O-P-O. Mientras que a 6 h se obtiene señales a 714 y $1082 \mathrm{~cm}^{-1}$ las cuales se relacionan al estiramiento $\mathrm{P}-\mathrm{O}$, estas regiones espectrales han sido previamente reportadas (Chambard, Marsan, \& Charvillat, 2019). Cuando el material no se trata térmicamente básicamente presenta las mismas señales que los demás espectros, pero son señales con más ruido que se podría atribuir a que la HAp posee una estructura amorfa. Lo cual pone de manifiesto, que la formación de la HAp cristalina es dependiente del tiempo. 

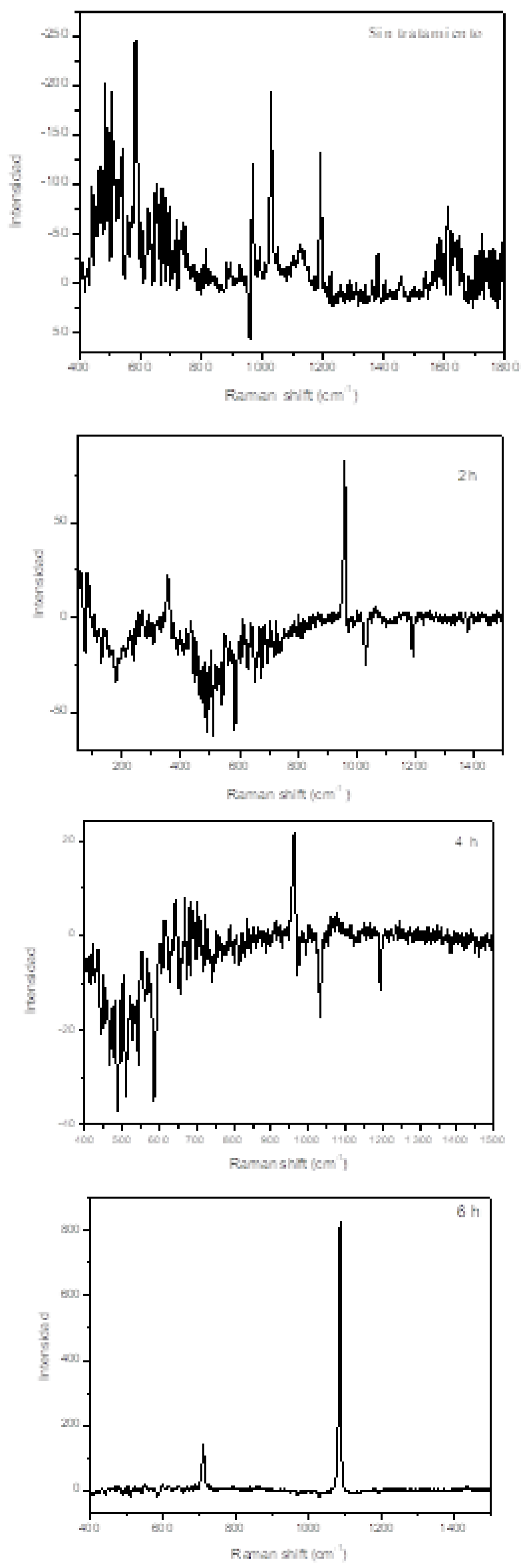

Figura 3 Espectro de HAp:) sin tratamiento, con tratamiento térmico: $2 \mathrm{hr}$, $4 \mathrm{hrs}$ y $6 \mathrm{hrs}$.

Fuente: Elaboración propia

\section{Conclusiones}

Es posible sintetizar HAp a partir del desecho del cascaron de huevo, un factor importante a controlar en este método, podría ser el tiempo de impregnación, y el tiempo del tratamiento térmico, en la relación $\mathrm{Ca} / \mathrm{P}$. En los espectros de FTIR y raman se encontró que el material obtenido presenta los grupos característicos de la HAp. Aunque se tienen valores mayores a 1.66, se obtienen una HAp carbonatada, lo cual puede ser beneficioso ya que estos materiales se quieren probar en la fotocatálisis para remoción de contaminantes.

\section{Referencias}

Akram, M., Ahmed, R., Shakir, I., Ibrahim, W., \& Hussain, R. (2014). Extracting hydroxyapatite and its precursors from natural resources. $J$ Mater Sci, 1461-1475.

Camacho Díaz, D., Vela, R., Villanueva, A., Borja, V., Montalvo, F., \& Quispe, M. (2018). Hidroxiapatita sintetizada a partir de la cáscara de huevo como potencial sustituto óseo en defectos periodontales y periimplantarios. Odontol. Sanmarquina, 296-301.

Chambard, M., Marsan, O., \& Charvillat, C. (2019). Effect of the deposition route on the microstructure of plasma-sprayed hydroxyapatite coatings. Surface and Coatings Technology, Elsevier, 68-77.

Coreño, J., Mújica, C., \& Hernández, C. (2010). Evaluación de hidroxiapatita nanoparticulada como material adsorbente de iones flúor, plomo y arsénico en soluciones acuosas. Superficies y Vacío, 161-165.

Dai, H., Tan, X., Zhu, H., Sun, T., \& Wang, X. (2018). Effects of Commonly Occurring Metal Ions on Hydroxyapatite Crystallization for Phosphorus Recovery fromWastewater. Water, $1-12$.

Fragoso, A., Vera, R., Pérez, G., Iglesias, A., Gómez, P., \& Villarreal, G. (2018). Síntesis y Caracterización de Hidroxiapatita Sintética para la Preparación de Filmes de PLGA/HAp con Potencial Uso en Aplicaciones Biomédicas. Biomédica, 93-116. 
García, G., \& Reyes, G. (2006). LA HIDROXIAPATITA, SU IMPORTANCIA EN LOS TEJIDOS. Revista Especializada en Ciencias Químico-Biológicas, 90-95.

García, S. (5 de Mayo de 2019). https://www.sinembargo.mx/05-052019/3573801. Obtenido https://www.sinembargo.mx/05-052019/3573801: https://www.sinembargo.mx/05$05-2019 / 3573801$

Gomes, D., Santos, A., Neves, G., \& Menezes, R. (2019). A brief review on hydroxyapatite production and use in biomedicine. Cerámica, 65(374), 282-302.

Jin, X., Chen, X., Cheng, Y., Wang, L., \& Hu, B. (2015). Effects of hydrothermal temperature and time on hydrothermal synthesis of colloidal hydroxyapatite nanorods in the presence of sodium citrate. Journal of Colloid and Interface Science, 151-158.

Londoño, M., Echavarría, A., \& De La Calle, F. (2006). características cristaloquímicas de la hidroxiapatita sintética tratada a diferentes temperaturas. EIA, 109-118.

Pleshko, N., Boskey , A., \& Mendelsoh, R. (1991). Novel infrared spectroscopic method for the determination of crystallinity of hydroxyapatite minerals. Biophys. J. $c$ Biophysical Society, 786-793.

Reyes, C. (2002). La Química Verde y la problemática de los residuos. Ciencia en Desarrollo, 131-146.

Rivera, J., Fetter, G., \& Bosch, P. (2011). Efecto del $\mathrm{pH}$ en la síntesis de hidroxiapatita en presencia de microondas. Revista Matéria, 506515.

Rivera, R., Riaño, H., Echavarría, A., Monsalve, P., Alzate, G., Restrepo, L., \& Jaramillo, C. (2004). Injertos óseos - Nueva alternativa. Fase III.Obtención, caracterización y evaluación de Hidroxiapatita Sintética y el compuesto de Hidroxiapatita Sintética porosa - Proteínas Morfogenéticas Óseas en un modelo experimental Lapino. Rev Col Cienc Pec , 20-28.
Ruíz, B., Reyes, L., Silva, H., Larrañaga, D. Estévez, M., \& Pérez, R. (2018). Novel biosynthesis of Ag-hydroxyapatite: Structural and spectroscopic characterization. ScienceDirect, 593-597.

Sánchez, G., Berini, A., \& Gay, E. (1993). LOS DIFERENTES TIPOS DE HIDROXIAPATITA Y SUS APLICACIONES EN LA CIRUGIA BUCAL. Avances en Odontoestomatología, 633-638. 